\title{
Association of serum concentrations of persistent organic pollutants with the prevalence of learning disability and attention deficit disorder
}

\author{
Duk-Hee Lee, David R Jacobs, Miquel Porta
}

J Epidemiol Community Health 2007;61:591-596. doi: 10.1136/jech.2006.054700 See end of article for
authors' affiliations

Correspondence to Dr D-H Lee, Department of Preventive Medicine, School of Medicine, Kyungpook University, 101 Dongindong, Jung-gu, Daegu 700422, Korea; lee_dh@knu.ac. $\mathrm{kr}$

Accepted 17November 2006

\begin{abstract}
Objective: Even though persistent organic pollutants (POPs) are well-known neurotoxicants, there is no previous study, even cross-sectional, on the association between background exposure to POPs and clinically significant developmental disorders, such as learning disability (LD) or attention deficit disorder (ADD), among children from a general population.

Design: Cross-sectional study.

Setting: Study subjects were 278 children aged 12-15 years included in the National Health and Nutrition Examination Survey 1999-2000. The seven most commonly detected POPs (each detectable in $\geqslant 20 \%$ of children: 3,3',4,4',5-pentachlorobiphenyl; 1,2,3,4,6,7,8-heptachlorodibenzo-p-dioxin (HPCDD); 1,2,3,4,6,7,8,9-octachlorodibenzo-p-dioxin (OCDD); 1,2,3,4,6,7,8-heptachlorodibenzofuran (HPCDF); $\beta$ hexachlorocyclohexane; $p, p^{\prime}$-dichlorodiphenyltrichloroethane; and trans-nonachlor) were selected.

Main results: Compared with children with non-detectable levels of POPs, adjusted prevalence ORs (95\% Cls) of $L D$ among those with detectable levels of HPCDD, OCDD or HPCDF were 2.08 ( 1.17 to 3.68 ), 2.72 (1.24 to 5.99 ) and 2.18 (1.15 to 4.15$)$, respectively. For $A D D$, the corresponding figures were 3.41 (1.08 to 10.8), 3.33 (0.94 to 11.8 ) and 2.31 (0.62 to 8.63 ), respectively.

Conclusions: Associations were observed between serum concentrations of POPs belonging to the categories of polychlorinated dibenzo-p-dioxins and polychlorinated dibenzofurans and the prevalence of two clinically significant development problems, LD and ADD. The nature of these associations needs to be clarified by prospective studies.
\end{abstract}

$E^{2}$ xtensive laboratory and clinical studies on several neurodevelopmental toxicants show the unique vulnerability of the developing brain to environmental chemical agents at low exposure levels that have little or no effect on adults. ${ }^{1} \mathrm{~A}$ number of prospective studies reported inverse associations between levels of various environmental neurotoxicants in mother's blood or cord blood and cognitive function during infancy or early childhood. ${ }^{2}$ However, some researchers question whether a slight decrease in cognitive function in early childhood, which is measured in continuous scales, can lead to the development of clinically significant developmental disorders. ${ }^{3}$ Evaluation of the clinical importance of the decrease in cognitive function found in prospective behavioural toxicology studies has been limited by the reluctance of investigators to set criteria to designate test score cut-offs that would be expected to reflect functionally significant deficits in the child's day-today function. ${ }^{3}$ In addition, to clarify the relationship between environmental neurotoxicants and the risk of developing clinically definite developmental disorders, very large cohort studies with a long follow-up are needed to observe a sufficient number of cases, owing to the low incidence of definite developmental disorders in general population.

As a contribution to investigate the association between background exposure to neurotoxicants and clinically significant developmental disorders, we compared the prevalence of developmental disorders among children with various concentrations of environmental neurotoxicants in a sample of the US general population. In the National Health and Nutrition Examination Survey (NHANES) 1999-2000, participants aged 4-15 years were asked whether they had a history of diagnosis of learning disability (LD) or attention deficit disorder (ADD), which are among childhood disabilities of increasing concern. ${ }^{45}$
Among several neurotoxic chemicals measured in NHANES, ${ }^{6}$ those with long half lives, including persistent organic pollutants (POPs), lead and cadmium, were our target environmental neurotoxicants. ${ }^{7-9}$ In the case of neurotoxicants with short half lives, current increased levels primarily reflect an increased exposure after developing LD or ADD; thus we did not consider them in our analyses.

POPs include hundreds of different organic chemical compounds with common properties such as long-term persistence, widespread diffusion in the environment and bioaccumulation in fatty tissues of living organisms. ${ }^{810-12} \mathrm{~A}$ growing body of scientific evidence associates human exposure to POPs with various health outcomes, including neurodevelopmental impairment through the disruption of the endocrine system. ${ }^{810}$ Even though the most problematic POPs were banned in many developed countries several decades ago, POP residues continue to be commonly found in animal feed and in the fat of fish, meat and dairy products, on a global scale. Humans are mainly exposed to POPs through fatty foods. ${ }^{811} 12$

Serum concentrations of lead and cadmium were measured in participants aged $\geqslant 1$ year, but POPs were measured only among those aged $\geqslant 12$ years. Thus, the associations of blood lead or cadmium with the prevalence of $\mathrm{LD}$ or $\mathrm{ADD}$ were

Abbreviations: $A D D$, attention deficit disorder; $A h R, A h$ receptor; $D D E$, $p, p^{\prime}$-dichlorodiphenyltrichloroethane; $\mathrm{HCH}, \beta$-hexachlorocyclohexane; HPCDD, 1, 2,3,4,6,7,8-heptachlorodibenzo-p-dioxin; HPCDF,

$1,2,3,4,6,7,8$-heptachlorodibenzofuran; LD, learning disability; LOD, limit of detection; NHANES, National Health and Nutrition Examination Survey; OCDD , 1,2,3,4,6,7,8,9-octachlorodibenzo-p-dioxin; PCB, pentachlorobiphenyl; POP, persistent organic pollutant; TEF, toxic equivalency factor; TNA, trans-nonachlor 
studied among children aged 4-15 years, whereas those of POPs were analysed among children aged $12-15$ years.

\section{METHODS}

\section{Study population}

The 1999-2000 NHANES (public-use dataset, http:// www.cdc.gov/nchs/about/major/nhanes/datalink.htm) conducted by the Centers for Disease Control and Prevention was designed to be nationally representative of the non-institutionalised, US civilian population on the basis of a complex, multistage probability sample. ${ }^{13}$

\section{Measurement}

Details of the NHANES protocol and procedures are available elsewhere. ${ }^{13}$ The NHANES standardised home interview was followed by a detailed physical examination in a mobile evaluation clinic or at the participant's home. A child was considered to have LD if the child or their parents reported that a representative from a school or a health professional ever said that the child had a LD. The child was considered to have ADD if there was a self-report that a doctor or health professional ever said that the child had ADD.

Venous blood and urine samples were collected and shipped every week at $-20^{\circ} \mathrm{C}$. Blood lead or cadmium levels were determined using graphite furnace atomic absorption spectrophotometry. A total of 53 biologically important POPs were measured as individual chemicals by high-resolution gas chromatography/high-resolution mass spectrometry using isotope dilution for quantification. All analytes were measured in approximately $5 \mathrm{ml}$ of serum using a modified method of Turner et al..$^{14}$ The POPs as provided by NHANES were adjusted for serum total cholesterol and triglycerides. ${ }^{15}$ It should be noted that these analyses have a limit of detection (LOD) partly related to the serum volume available for measuring POPs, which was slightly different depending on how much blood each subject provided. Only the maximum observed LOD for each POP was provided by NHANES. A higher sample volume results in a lower LOD and a better ability to detect lower concentrations of POPs.

We did not study all 53 POPs because most children had serum concentrations of many POPs below the LOD. We therefore selected seven compounds for which at least $20 \%$ of study subjects had concentrations above the LOD: 3,3',4,4',5pentachlorobiphenyl (PCB126); 1,2,3,4,6,7,8-heptachlorodibenzo- $p$-dioxin (HPCDD); 1,2,3,4,6,7,8,9-octachlorodibenzo- $p$ dioxin (OCDD); 1,2,3,4,6,7,8-heptachlorodibenzofuran (HPCDF); $\beta$-hexachlorocyclohexane $(\mathrm{HCH}) ; \quad \mathrm{p}, \mathrm{p}^{\prime}$-dichlorodiphenyltrichloroethane (DDE); and trans-nonachlor (TNA).

\section{Final samples}

Among the 2735 children aged 4-15 years, 2252 had information on blood lead and among the 1251 children aged 1215 years, 279 had information on all seven POPs because POPs were measured only in a sub-sample. Owing to missing information on the history of LD or ADD, the final sample sizes were 2246 and 278 children, respectively. As the epidemiologically relevant findings were observed only with POPs, we mention the findings for 2246 children related to lead and cadmium as an important null finding, but we focus primarily on the association between serum concentrations of POPs and LD or ADD among the 278 children.

\section{Statistical methods}

Blood lead levels were classified into quintiles; cut-off points of blood lead quintiles were $0.8,1.2,1.7$ and $2.4 \mu \mathrm{g} / \mathrm{dl}$. However, in the case of blood cadmium, $52.7 \%$ had concentration of $0.2 \mu \mathrm{g} /$ $\mathrm{dl}, 20.7 \%$ had $0.3 \mu \mathrm{g} / \mathrm{dl}$ and $20.1 \%$ had $0.4 \mu \mathrm{g} / \mathrm{dl}$. Thus blood concentrations of cadmium were divided into four groups: 0.2, $0.3,0.4$ and $\geqslant 0.5 \mu \mathrm{g} / \mathrm{dl}$. For each POP, we also compared subjects with serum concentrations below the LOD and subjects with detectable levels. The exception was DDE, detectable in all participants and split at its median.

Logistic regression models were used to calculate multivariate-adjusted prevalence odds ratios (ORs). Adjusting variables were sex, race/ethnicity, age (years), poverty:income ratio (continuous, wealthier people having higher values), birth weight (continuous), mother's age at birth (continuous), cigarette smoking during pregnancy (yes/no), body mass index (continuous) and saturated fat intake (continuous). All statistical analyses were performed with SAS V.9.1 and SUDAAN V.9.0. Estimates of main results were calculated to account for stratification and clustering, ${ }^{16}$ adjusting for age, race/ethnicity, and poverty:income ratio instead of using sample weights; this adjustment is regarded as a good compromise between efficiency and bias. ${ }^{16}{ }^{17}$

\section{RESULTS}

Table 1 shows the distribution of potential confounding variables by LD and ADD among the 278 children. Children with LD or ADD were more likely to be white, with young mothers, who were more likely to have smoked during pregnancy.

Neither blood lead nor cadmium concentrations were associated with LD or ADD in the 278 children in whom POPs were measured. In the larger sample of 2246 children in whom lead and cadmium were also measured, the adjusted ORs for blood lead (highest vs lowest quintiles) were 0.86, 0.88, 1.09 and 1.25 for LD ( $p$ value for trend $=0.12$ ) and 1.04, 0.77, 1.12 and 0.87 for ADD ( $p$ value for trend $=0.80$ ). The corresponding figures for blood cadmium were $0.82,1.02$ and 1.02 for $\mathrm{LD}(\mathrm{p}$ value for trend $=0.96$ ) and $1.13,1.27$ and 1.44 for $\mathrm{ADD}(\mathrm{p}$ value for trend $=0.27$ ), respectively; this nonsignificant positive trend disappeared after additional adjustment for POPs.

Among the seven POPs, HPCDD, OCDD and HPCDF showed significant positive associations with the prevalence of LD (table 2). After adjustment for age, race/ethnicity, sex, poverty index ratio, mother's age at pregnancy, birth weight, body mass index and saturated fat intake, compared with children with non-detectable levels of POPs, ORs (95\% CIs) among those with detectable levels of HPCDD, OCDD and HPCDF were 2.08 ( 1.17 to 3.68$), 2.72$ ( 1.24 to 5.99 ) and 2.18 ( 1.15 to 4.15 ), respectively. In contrast, PCB126, DDE and TNA were not associated with $\mathrm{LD}$; $\mathrm{HCH}$ was inversely, but not significantly, associated with LD: the adjusted OR (95\% CI) was 0.37 ( 0.13 to 1.02 ). Additional adjustment for blood lead and cadmium did not materially change the results.

The associations of POPs with ADD were similar to those with LD, although most POPs did not reach statistical significance (table 3); adjusted ORs (95\% CIs) for HPCDD, OCDD and HPCDF were 3.41 ( 1.08 to 10.8 ), 3.33 (0.94 to 11.8 ) and 2.31 (0.62 to 8.63), respectively. Similar to LD, the association with $\mathrm{HCH}$ was inverse, but not close to statistical significance.

\section{DISCUSSION}

A number of prospective studies ${ }^{18-25}$ have reported that prenatal exposure to background POPs could impair intellectual and/or behavioural development among infants and toddlers; however, findings have not been totally consistent whether these effects persist among children. Some studies reported a harmful effect of prenatal exposure to POPs on cognitive and motor abilities even among school-aged children, ${ }^{26}{ }^{27}$ but other studies reported that the detrimental effects of POPs are only transient 
Table 1 Distribution of child-and family-related potential confounding factors according to the presence of learning disability or attention deficit disorder

\begin{tabular}{|c|c|c|c|c|c|c|}
\hline & \multicolumn{3}{|c|}{ Learning disability } & \multicolumn{3}{|c|}{ Aftention deficit disorder } \\
\hline & No $(n=234)$ & Yes $(n=44)$ & $\mathrm{p}$ Value & No $(n=251)$ & Yes $(n=26)$ & P Value \\
\hline \multicolumn{7}{|l|}{ Children's variables } \\
\hline Age (years) & $13.4(1.0)$ & $13.2(1.1)$ & 0.19 & $13.4(1.1)$ & $13.0(1.0)$ & 0.06 \\
\hline Male (\%) & 48.7 & 63.6 & 0.07 & 50.2 & 57.7 & 0.47 \\
\hline White (\%) & 17.5 & 43.2 & $<0.01$ & 18.3 & 53.8 & $<0.01$ \\
\hline Birth weight (g) & 3288 (682) & $3151(709)$ & 0.23 & $3293(660)$ & 3049 (879) & 0.18 \\
\hline Body mass index & $23.1(5.4)$ & $22.3(4.5)$ & 0.37 & $22.9(5.1)$ & $23.3(6.9)$ & 0.79 \\
\hline Saturated fat intake (g) & $27.5(14.7)$ & $26.9(17.1)$ & 0.81 & $27.0(15.1)$ & $31.4(14.5)$ & 0.16 \\
\hline Blood lead $(\mu \mathrm{g} / \mathrm{dll})$ & $1.6(1.3)$ & $1.6(0.9)$ & 0.79 & $1.6(1.2)$ & $1.8(1.4)$ & 0.41 \\
\hline Blood cadmium $(\mu \mathrm{g} / \mathrm{l})$ & $0.3(0.1)$ & $0.3(0.2)$ & 0.67 & $0.3(0.2)$ & $0.3(0.2)$ & 0.86 \\
\hline \multicolumn{7}{|l|}{ Family and maternal variables } \\
\hline Poverty:income ratio & $1.8(1.3)$ & $1.7(1.5)$ & 0.66 & $1.8(1.4)$ & $1.4(1.4)$ & 0.22 \\
\hline Mother's age at pregnancy (years) & $25.0(6.1)$ & $23.6(5.7)$ & 0.17 & $25.1(6.1)$ & $21.9(4.6)$ & 0.01 \\
\hline $\begin{array}{l}\text { Cigarette smoking during } \\
\text { pregnancy (\%) }\end{array}$ & $15.0^{\circ}$ & $40.9^{\circ}$ & $<0.01$ & 16.7 & 42.3 & 0.02 \\
\hline
\end{tabular}

or observed only among children raised in suboptimal environments. ${ }^{28}{ }^{29}$ Moreover, whether this exposure is associated with clinically significant developmental disorders such as $\mathrm{LD}$ or $\mathrm{ADD}$, rather than with continuous scores of intellectual or behavioural functions among school-aged children has never been studied. Slightly decreased scores of cognitive function do not necessarily mean the presence of clinically significant developmental disorders. ${ }^{3}$
In this study, serum concentrations of polychlorinated dibenzo-p-dioxins (PCDDs) and polychlorinated dibenzofurans (PCDFs) were positively associated with the prevalence of LD or ADD among children aged 12-15 years. Our findings are basically consistent with those of prospective cohort studies, which showed decreased scores of intellectual or behavioural functions after low-level exposures to some POPs during pregnancy or early infancy. These findings are also biologically

Table 2 Adjusted* prevalence OR and $95 \% \mathrm{Cl}$ of learning disability by category of seven persistent organic pollutants

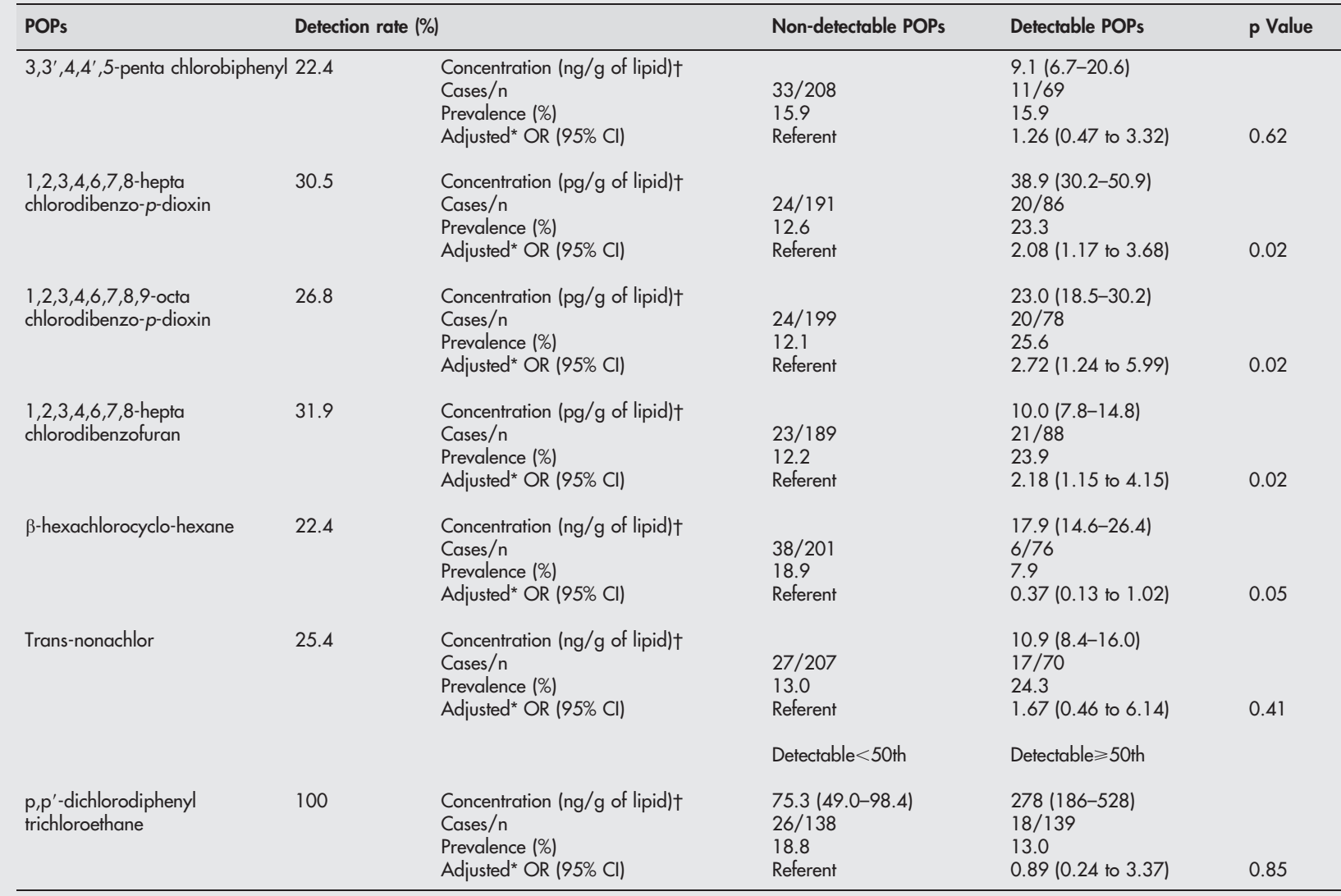

POP, persistent organic pollutant.

*Adjustment for age, race/ethnicity, sex, poverty index ratio, mother's age at pregnancy, cigarette smoking during pregnancy, birth weight, body mass index and saturated fat intake.

†Median (interquartile range). 
Table 3 Adjusted* prevalence OR and $95 \% \mathrm{Cl}$ of attention deficit disorder by category of seven persistent organic pollutants

\begin{tabular}{|c|c|c|c|c|c|}
\hline POPs & Detection rate $(\%)$ & & Non-detectable POPs & Detectable POPs & p Value \\
\hline $3,3^{\prime}, 4,4^{\prime}, 5$-penta chlorobiphenyl & 22.4 & $\begin{array}{l}\text { Concentration (ng/g of lipid)† } \\
\text { Cases/n } \\
\text { Prevalence (\%) } \\
\text { Adjusted* OR (95\% Cl) }\end{array}$ & $\begin{array}{l}20 / 208 \\
9.6 \\
\text { Referent }\end{array}$ & $\begin{array}{l}9.1(6.7-20.6) \\
6 / 69 \\
8.7 \\
1.03(0.37 \text { to } 2.88)\end{array}$ & 0.96 \\
\hline $\begin{array}{l}\text { 1,2,3,4,6,7,8-hepta } \\
\text { chlorodibenzo-p-dioxin }\end{array}$ & 30.5 & $\begin{array}{l}\text { Concentration (pg/g of lipid) } \dagger \\
\text { Cases } / \mathrm{n} \\
\text { Prevalence }(\%) \\
\text { Adjusted* OR }(95 \% \mathrm{Cl})\end{array}$ & $\begin{array}{l}12 / 191 \\
6.3 \\
\text { Referent }\end{array}$ & $\begin{array}{l}38.9(30.2-50.9) \\
14 / 86 \\
16.3 \\
3.41 \text { (1.08 to } 10.8)\end{array}$ & 0.04 \\
\hline $\begin{array}{l}1,2,3,4,6,7,8,9- \\
\text { octachlorodibenzo-p-dioxin }\end{array}$ & 26.8 & $\begin{array}{l}\text { Concentration (pg/g of lipid)† } \\
\text { Cases } / \mathrm{n} \\
\text { Prevalence (\%) } \\
\text { Adjusted* OR ( } 95 \% \text { Cl) }\end{array}$ & $\begin{array}{l}12 / 199 \\
6.0 \\
\text { Referent }\end{array}$ & $\begin{array}{l}23.0(18.5-30.2) \\
14 / 78 \\
18.0 \\
3.33(0.94 \text { to } 11.8)\end{array}$ & 0.06 \\
\hline $\begin{array}{l}\text { 1,2,3,4,6,7,8-hepta } \\
\text { chlorodibenzofuran }\end{array}$ & 31.9 & $\begin{array}{l}\text { Concentration (pg/g of lipid)† } \\
\text { Cases } / \mathrm{n} \\
\text { Prevalence (\%) } \\
\text { Adjusted* OR ( } 95 \% \mathrm{Cl})\end{array}$ & $\begin{array}{l}13 / 189 \\
6.9 \\
\text { Referent }\end{array}$ & $\begin{array}{l}10.0(7.8-14.8) \\
13 / 88 \\
14.8 \\
2.31(0.62 \text { to } 8.63)\end{array}$ & 0.20 \\
\hline$\beta$-hexachlorocyclo hexane & 22.4 & $\begin{array}{l}\text { Concentration (ng/g of lipid)† } \\
\text { Cases/n } \\
\text { Prevalence (\%) } \\
\text { Adjusted* OR ( } 95 \% \text { Cl) }\end{array}$ & $\begin{array}{l}23 / 201 \\
11.4 \\
\text { Referent }\end{array}$ & $\begin{array}{l}17.9(14.6-26.4) \\
3 / 76 \\
4.0 \\
0.44(0.08 \text { to } 2.62)\end{array}$ & 0.34 \\
\hline Trans-nonachlor & 25.4 & $\begin{array}{l}\text { Concentration (ng/g of lipid)† } \\
\text { Cases } / \mathrm{n} \\
\text { Prevalence (\%) } \\
\text { Adjusted* OR ( } 95 \% \text { Cl) }\end{array}$ & $\begin{array}{l}17 / 207 \\
8.2 \\
\text { Referent } \\
\text { Detectable }<50 \text { th }\end{array}$ & $\begin{array}{l}10.9(8.4-16.0) \\
9 / 70 \\
12.9 \\
1.58(0.57 \text { to } 4.42) \\
\text { Detectable } \geqslant 50 \text { th }\end{array}$ & 0.35 \\
\hline $\begin{array}{l}\text { p,p'-dichlorodiphenyl } \\
\text { trichloroethane }\end{array}$ & 100 & $\begin{array}{l}\text { Concentration (ng/g of lipid) } \dagger \\
\text { Cases/n } \\
\text { Prevalence (\%) } \\
\text { Adjusted* OR }(95 \% \text { Cl) }\end{array}$ & $\begin{array}{l}75.3(49.0-98.4) \\
17 / 138 \\
12.3 \\
\text { Referent }\end{array}$ & $\begin{array}{l}278(186-528) \\
9 / 139 \\
6.5 \\
1.25(0.30 \text { to } 5.26)\end{array}$ & 0.74 \\
\hline
\end{tabular}

POP, persistent organic pollutant.

*Adjustment for age, race/ethnicity, sex, poverty index ratio, mother's age at pregnancy, birth weight, cigarette smoking during pregnancy, body mass index and saturated fat intake.

†Median (interquartile range).

plausible because POPs are well-known neurotoxicants, even at low doses. ${ }^{10}{ }^{30}$ It may seem surprising that there has been no previous study, even cross-sectional, with the outcome of clinically significant developmental disorders. The absence of such studies probably reflects the practical difficulty that measurements of serum concentrations of POPs require such amounts of serum which are large for children.

Cross-sectional findings may reflect reverse causality. Thus, it is important to evaluate whether serum concentrations of POPs can increase after development of LD or ADD. Children with low body mass index or weight loss may experience an increase in serum concentrations of POPs due to bioconcentration. ${ }^{31} 32$ Thus, if changes in body weight occur after development of LD or ADD, the positive cross-sectional association may be due to reverse causality. However, in this study population, body mass index was not associated with the presence of LD or ADD and body mass index was adjusted for in the final analyses. As there was no information on changes in body weight among children, it was impossible to directly evaluate the effect of weight loss. However, if the release of POPs from adipose tissue due to weight loss were a problem in analysing the association between POPs and LD or ADD, all POPs contained in the adipose tissue would have been positively associated, whereas only PCDDs and PCDFs, not PCBs or organochlorine pesticides, were positively associated with the prevalence of LD or ADD. Taken together, we think that neither high body mass index nor weight loss explain our findings. As POPs in the general population, except breastfeeding infants, come from animal products (eg, meat, milk, eggs and fish), ${ }^{34}$ a positive association might also be observed if intake of animal products increased sharply after development of LD or ADD. However, the current intake of saturated fat (used as a marker of intake of animal products) was not different between children with LD or ADD and those without either condition. This suggests that the exposure to POPs did not increase due to more intake of animal products after development of LD or ADD, thereby further reducing the possibility of reverse causality. In addition, some studies have reported that serum POPs levels were most strongly determined by breast feeding even in school-aged children, not by their diet. ${ }^{32} 33$

As serum concentrations of some POPs have been decreasing over several decades in the US general population, ${ }^{35}$ the current increase in LD or ADD may seem to be contradictory. However, similar to our earlier observation concerning the influence of obesity and POPs on the risk of type 2 diabetes, ${ }^{36}$ other factors that have recently increased may interact with POPs on the risk of LD or ADD. Alternatively, other POPs which were not measured in NHANES dataset, like the brominated flame retardants, perfluorinated compounds or chlorinated paraffins, which are increasingly used in the US and also show neurotoxicity in experimental studies, ${ }^{37} 38$ may be important and correlated with POPs measured in NHANES.

Most POPs were non-detectable among the children included in this study, and even common POPs were detected only in about $20 \%$ of children. We suspect that this limited detection is primarily due to limited amounts of serum used for POPs measurement. Most available evidence indicates that POPs would have been detectable in almost all children if sufficient 
amounts of serum had been used. ${ }^{12}$ In fact, concentrations of different POPs probably fluctuate differentially over time in different geographical areas. ${ }^{12} 39$

Most epidemiological studies have studied the extent of postnatal exposure through indirect measurements of POPs in breast milk among breastfeeding infants. Unlike prenatal exposures, postnatal exposures to POPs through breast milk were mostly not associated with cognitive function among infants or children, ${ }^{18} 192324$ with some exceptions. ${ }^{10}{ }^{40}$ Exposure through breast milk may differ from that through other foodstuffs because breast milk is believed to have many beneficial effects for infant development. ${ }^{41}$ POPs concentrations in children aged 12-15 years may be influenced by animal products consumption as well as breast milk. Postnatal exposure to environmental neurotoxicants through animal products may be harmful. Or, the current study might suggest that exposure to POPs contained in breast milk may present adverse effects in the long term. Although the most serious effects of neurotoxicants seem to be on cell migration and differentiation (processes that are most active prenatally), brain development continues at a slower pace for years postnatally. ${ }^{42}$

A class of hexachlorocyclohexane is a well-known neurotoxin, although most experimental studies have been performed with $\gamma$-hexachlorocyclohexane. ${ }^{43}$ Thus, the inverse associations of $\mathrm{HCH}$ with LD or ADD may be strange. We think that these inverse associations may be due to complicated interactions among chemical mixtures with similar pathways when simultaneous exposure occurs. Toxicological studies have reported that potentiation, inhibition, antagonism, synergism or other interactions of chemicals in an organism may significantly affect the toxicity of chemical mixtures. ${ }^{44}$ Alternatively, they may be false positive findings.

The concept of toxic equivalency factors (TEFs), a measure of the ability to bind to the Ah receptor (AhR), has been developed to facilitate risk assessment and regulatory control of exposure to complex POPs mixtures. ${ }^{45}$ However, we did not use TEFs to calculate the cumulative effect of POPs because the strength of association of the POPs observed in this study did not seem to be correlated with the respective TEFs, leading us to hypothesise that binding to AhR may not be the critical pathway. For example, the TEFs of HPCDD and OCDD, 0.01 and 0.0001 , were not concordant with their LD ORs of 2.08 and 2.72, respectively (table 2). Supporting this speculation, there is evidence that PCB congeners have differential effects on end points of neurotoxicity depending on their chemical structure: specifically, the ortho-substituted congeners which have low affinity for the AhR are neurotoxic, whereas coplanar (dioxin-like) congeners are relatively inactive in producing neurotoxic effects. ${ }^{46}$

In contrast, even though many cross-sectional or prospective studies have reported associations between blood lead levels and neurodevelopmental deficits in school-aged children, ${ }^{47-49}$ blood lead levels were not associated with the presence of LD or ADD in this study. The more important point may be that the $97 \%$ of the study subjects had blood lead levels $<5 \mu \mathrm{g} / \mathrm{dl}$. Even though these levels of blood lead could be related to subtle intellectual impairment as shown in previous studies ${ }^{47}$ they may not lead to the development of definite developmental disorders. Besides lead, there are only a few studies on the association between cadmium exposure and cognitive function in children. ${ }^{50}{ }^{51}$ In this study, blood cadmium levels showed a weak positive trend with ADD, but it disappeared after adjusting for POPs. Similar to our findings, a Dutch study also reported a positive association of cognitive abilities with POPs, but not with lead or cadmium. ${ }^{29}$

This study has several limitations. First, the cross-sectional study design. Second, LD or ADD was self-reported, probably
What this paper adds

- Persistent organic pollutants (POPs) are well-known neurotoxicants. Prospective cohort studies have reported lower scores for continuous markers of intellectual or behavioural functions after low-level exposures to some POPs.

- No previous study, not even of cross-sectional design, has examined whether there is an association between background exposure to POPs and clinically significant developmental disorders, such as learning disability (LD) or attention deficit disorder (ADD), in the general population.

- This cross-sectional study found that children who had high serum concentrations of POPs belonging to the categories of polychlorinated dibenzo-p-dioxins and polychlorinated dibenzofurans showed about twice the prevalence of $L D$ or $A D D$ as those with non-detectable levels.

\section{Policy implications}

Developmental disorders such as learning disabilities or attention deficit disorders have a significant impact on community resources. As consumption of animal products is the main source of background exposure to PCDDs or PCDFs in general population, POPs may be a preventable and treatable cause of developmental disorders.

including children who were having any of a broad range of problems. Use of objective diagnostic criteria for LD or ADD may be feasible in clinical settings. However, in epidemiological studies among general populations, self-reported disease outcomes may be informative, even if misclassification leads to the underestimation of true relationships. In addition, in this study, the results were not different for LD and ADD. These points suggest that background exposure to POPs may be associated with a broad range of developmental disorders among children. Third, although some subjects with a higher value of POP but a lower sample volume might have been classified in the reference group, and vice versa, such misclassifications would be unrelated to self-reported LD or ADD, again weakening the true strength of association. Fourth, we could only adjust for variables that were examined in NHANES and there could be residual confounding. Fifth, we could not evaluate other pollutants such as methylmercury as it was not measured among children aged $4-15$ years.

In summary, there were positive relationships between serum concentrations of selected POPs and the prevalence of two clinically relevant developmental problems, LD and ADD. A possible role of background exposure to POPs in future risk of developmental disorder needs to be clarified in large prospective studies, conducted in the general population.

\section{Authors' affiliations}

Duk-Hee Lee, Department of Preventive Medicine and Health Promotion Research Center, School of Medicine, Kyungpook National University, Daegu, Korea

David R Jacobs, Division of Epidemiology, School of Public Health, University of Minnesota, Minnesota, USA

Miquel Porta, Institut Municipal d'Investigació Mèdica, Barcelona, Spain

Competing interests: None declared. 


\section{REFERENCES}

1 Schettler T. Toxic threats to neurologic development of children. Environ Health Perspect 2001:109:813-16.

2 Trask CL, Kosofsky BE. Developmental considerations of neurotoxic exposures. Neurol Clin 2000;18:541-62.

3 Jacobson JL, Jacobson SW. Methodological issues in research on developmental exposure to neurotoxic agents. Neurotoxicol Teratol 2005;27:395-406.

4 Scahill L, Schwab-Stone M. Epidemiology of ADHD in school-age children. Child Adolesc Psychiatr Clin N Am 2000;9:541-55.

5 Halfon N, Newacheck PW. Prevalence and impact of parent-reported disabling mental health conditions among U.S. children. J Am Acad Child Adolesc Psychiatry 1999;38:600-9.

6 Department of Health and Human Services, Centers for Disease Control and Prevention, National Center for Environmental Health. Third National Report on Human Exposure to Environmental Chemicals. NECH publication No 05-0570. Atlanta: Centers for Disease Control and Prevention, 2005, Available at:http:// www.cdc.gov/exposurereport/3rd/pdf/thirdreport.pdf (accessed 17 Apr 2007)

7 Jarup, $M$ Berglund, CG Elinder, et al. Health effects of cadmium exposure-a review of the literature and a risk estimate. Scand J Work Environ Health 1998;24:1-51.

8 DeVito MJ, Birnbaum LS, Farland WH, et al. Comparisons of estimated human body burdens of dioxin-like chemicals and TCDD body burdens in experimentally exposed animals. Environ Health Perspect 1995; 103:820-31.

9 Tong S, von Schirnding YE, Prapamontol T. Environmental lead exposure: a public health problem of global dimensions. Bull World Health Organ 2000;78:1068-77.

10 Ribas-Fito N, Sala M, Kogevinas M, et al. Polychlorinated biphenyls (PCBs) and neurological development in children: a systematic review. I Epidemiol Community Health 2001;55:537-46.

11 Schafer KS, Kegley SE. Persistent toxic chemicals in the food supply. J Epidemiol Community Health 2002;56:813-17.

12 Porta M. Persistent toxic substances: exposed individuals and exposed populations. J Epidemiol Community Health 2004;58:534-5.

13 NHANES 1999-2000 public data release file documentation (article online). Available at: http://www.cdc.gov/nchs/about/major/nhanes/ nhanes99_00.htm (accessed 17 Apr 2007).

14 Turner W, DiPietro E, Lapeza C, et al. A fast universal automated cleanup system for the isotope-dilution HRMS analysis of PCDDs, PCDFs, coplanar PCBs, PCB congeners, and persistent pesticides from the same serum sample. Organohalogen Comp 1997;31:26-31.

15 NHANES 1999-2000 laboratory procedure manual. Available at: hitp:// www.cdc.gov/nchs/data/nhanes/frequency//28poc_b_met_pcb_pesticides.pdf (accessed 17 Apr 2007)

16 Korn EL, Graubard BI. Epidemiologic studies utilizing surveys: accounting for the sampling design. Am J Public Health 1991;81:1166-73.

17 Graubard BI, Korn EL. Analyzing health surveys for cancer-related objectives. $J$ Natl Cancer Inst 1999;91:1005-16.

18 Gladen BC, Rogan WJ, Hardy P, et al. Development after exposure to polychlorinated biphenyls and dichlorodiphenyl dichloroethene transplacentally and through human milk. J Pediatr 1988;113:991-5.

19 Rogan WJ, Gladen BC. PCBs, DDE, and child development at 18 and 24 months. Ann Epidemiol 1991;1:407-13.

20 Jacobson JL, Jacobson SW, Humphrey HE. Effects of in utero exposure to polychlorinated biphenyls and related contaminants on cognitive functioning in young children. J Pediatr 1990;116:38-45.

21 Darvill T, Lonky E, Reihman J, et al. Prenatal exposure to PCBs and infant performance on the Fagan test of infant intelligence. Neurotoxicology 2000:21:1029-38.

22 Huisman M, Koopman-Esseboom C, Fidler V, et al. Perinatal exposure to polychlorinated biphenyls and dioxins and its effect on neonatal neurological development. Early Hum Dev 1995;41:111-27.

23 Koopman-Esseboom C, Weisglas-Kuperus N, de Ridder MA, et al. Effects of polychlorinated biphenyl/dioxin exposure and feeding type on infants' mental and psychomotor development. Pediatrics 1996;97:700-6.

24 Patandin S, Lanting $\mathrm{Cl}$, Mulder PG, et al. Effects of environmental exposure to polychlorinated biphenyls and dioxins on cognitive abilities in Dutch children at 42 months of age. J Pediatr 1999:134:33-41.

25 Nakajima S, Saijo Y, Kato S, et al. Effects of prenatal exposure to polychlorinated biphenyls and dioxins on mental and motor development in
Japanese children at 6 months of age. Environ Health Perspect 2006;114:773-8.

26 Jacobson JL, Jacobson SW. Intellectual impairment in children exposed to polychlorinated biphenyls in utero. N Engl J Med 1996;335:783-9.

27 Vreugdenhil HJ, Mulder PG, Emmen HH, et al. Effects of perinatal exposure to PCBs on neuropsychological functions in the Rotterdam cohort at 9 years of age. Neuropsychology 2004;18:185-93.

28 Gladen BC, Rogan WJ. Effects of perinatal polychlorinated biphenyls and dichlorodiphenyl dichloroethene on later development. J Pediatr 1991;119:58-63.

29 Vreugdenhil $\mathrm{HJ}$, Lanting $\mathrm{Cl}$, Mulder PG, et al. Effects of prenatal PCB and dioxin background exposure on cognitive and motor abilities in Dutch children at schoo age. J Pediatr 2002;140:48-56.

30 Boersma ER, Lanting $\mathrm{Cl}$. Environmental exposure to polychlorinated biphenyls (PCBs) and dioxins. Consequences for longterm neurological and cognitive development of the child lactation. Adv Exp Med Biol 2000;478:271-87.

31 Chevrier J, Dewailly E, Ayotte P, et al. Body weight loss increases plasma and adipose tissue concentrations of potentially toxic pollutants in obese individuals. Int J Obes Relat Metab Disord 2000;24:1272-8.

32 Karmaus W, DeKoning EP, Kruse $\mathrm{H}$, et al. Early childhood determinants of organochlorine concentrations in school-aged children. Pediatr Res 2001;50:331-6.

33 Schantz SL, Jacobson JL, Humphrey HE, et al. Determinants of polychlorinated biphenyls (PCBs) in the sera of mothers and children from Michigan farms with PCB-contaminated silos. Arch Environ Health 1994;49:452-8.

34 Charnley G, Doull J. Human exposure to dioxins from food, 1999-2002. Food Chem Toxicol 2005:43:671-9.

35 Needham LL, Barr DB, Caudill SP, et al. Concentrations of environmental chemicals associated with neurodevelopmental effects in U.S. population. Neurotoxicology 2005;26:531-45

36 Lee DH, Lee IK, Song K, et al. A strong dose-response relation between serum concentrations of persistent organic pollutants and diabetes: results from the national health and examination survey 1999-2002. Diabetes Care 2006;29:1638-44.

37 Mariussen E, Fonnum F. Neurochemical targets and behavioral effects of organohalogen compounds: an update. Crit Rev Toxicol 2006:36:253-89.

38 Branchi I, Capone F, Alleva E, et al. Polybrominated diphenyl ethers: neurobehavioral effects following developmental exposure. Neurotoxicology 2003;24:449-62

39 Solomon GM, Weiss PM. Chemical contaminants in breast milk: time trends and regional variability. Environ Health Perspect 2002;1 10:339-47.

40 Walkowiak J, Wiener JA, Fastabend A, et al. Environmental exposure to polychlorinated biphenyls and quality of the home environment: effects on psychodevelopment in early childhood. Lancet 2001;358:1602-7.

41 Schanler RJ, Hurst NM, Lau C. The use of human milk and breasffeeding in premature infants. Clin Perinatol 1999:26:379-98.

42 Ornoy A. The impact of intrauterine exposure versus postnatal environment in neurodevelopmental toxicity: long-term neurobehavioral studies in children at risk for developmental disorders. Toxicol Lett 2003;140-141:171-81.

43 Singal A, Thami GP. Lindane neurotoxicity in childhood. Am J Ther 2006;13:277-80.

44 Cassee FR, Groten JP, van Bladeren PJ, et al. Toxicological evaluation and risk assessment of chemical mixtures. Crit Rev Toxicol 1998;28:73-101.

45 van den Berg M, Peterson RE, Schrenk D. Human risk assessment and TEFs. Food Addit Contam 2000; 17:347-58

46 Tilson HA, Kodavanti PR, Mundy WR, et al. Neurotoxicity of environmental chemicals and their mechanism of action. Toxicol Lett 1998;102-103:631-5.

47 Pocock SJ, Smith M, Baghurst P. Environmental lead and children's intelligence: a systematic review of the epidemiological evidence. BMJ 1994;309:1189-97.

48 Lanphear BP, Hornung R, Khoury J, et al. Low-level environmental lead exposure and children's intellectual function: an international pooled analysis. Environ Health Perspect 2005;113:894-9.

49 Canfield RL, Henderson CR Jr, Cory-Slechta DA, et al. Intellectual impairment in children with blood lead concentrations below $10 \mu \mathrm{g}$ per deciliter. N Engl J Med 2003;348:1517-26.

50 Thatcher RW, Lester ML, McAlaster R, et al. Effects of low levels of cadmium and lead on cognitive functioning in children. Arch Environ Health 1982;37:159-66.

51 Lewis $\mathbf{M}$, Worobey J, Ramsay DS, et al. Prenatal exposure to heary metals: effect on childhood cognitive skills and health status. Pediatrics 1992;89:1010-15. 